\section{A CLINICAL STUDY OF MULTIPLE NEURITIS IN YOUNG CHILDREN.}

Precented to the Section on Diseases of Children, at the Forty-ninth Annual Meeting of the American Medical Association, held at Denver, Colo., June i-10, 1898.

BY ANNE STURGIS DANIEL.

PROFESSOR OF PEDITRICS WOMAN'S MEDTCAL COLLEGE OF THE NEW YORK INFIRMARY.

$$
\text { NEW YORK CITY }
$$

The following brief clinical studies of multiple neuritis embrace only those cases in which were found motor and sensory paralysis of symmetric development, but do not include that form of neuritis which affects only the muscles of the palate or of the nose, as shown by regurgitations of fluids, with nasal voice or those cases affecting only the ocular muscles.

Diphtheria is undoubtedly by far the greatest cause of multiple neuritis. Comparatively few cases are caused by the other infectious diseases. Smallpox is not infrequently a cause of the disease, but I have seen no mention made of chicken-pox giving rise to the disease. I, unfortunately, have one case to report of the production of the disease by arsenic given during the course of treatment of chorea. I have twelve cases to report, the causes of which were: arsenic administered for chorea, 1 child; diphtheria, 9 cases; varicella, 1 case, and measles 1 case. Seven children were cured, 5 died. In none of my cases were the sphincters involved; in all cases there were some deviations from the normal mental condition of the child. This last symptom I have seen little stress laid upon. The first symptom noticed was simply a weakness of the extremities, followed rapidly by inability to stand or walk - the motor paralysis appearing first, followed at once by the sensory paralysis, the recovery being first of the sensory and later of the riotor paralysis.

No allusion was made in medical literature of paralysis following diphtheria until the end of the 16th century, the first mention being made in 1580 . Ghisi, in 1747, describes an attack in his own son, and adds that he "left to nature the cure of the strange consequences." The first description of the disease in America was given by Dr. Bard of New York, in 1771 . Few authorities describe multiple neuritis in children, although diphtheretic paralysis was described long before the disease was considered to be a neuritis. In looking over the statistics of paralysis following diphtheria, I find no distinction is made, or in only a few instances, of paralysis affecting the throat only and a general multiple paralysis. In the collective investigation of the American Pediatric Society, of 189 cases, the paralysis was general in 8 only. They report 3384 cases with 9.7 per cent. followed by paralysis. Various other estimates are given of from 5 to 15 per cent. All authorities agree as to the rarity of death, although one authority thinks physicians are too optimistic in regard to the mortality. Of my 9 cases, 3 were treated during the attack of diphtheria with antitoxin; of these three, 2 died. Five of the 9 children were treated during the attackof diphtheria In 7 cases the Klebs-Loeffler bacilli were found; the other two occurred before the discovery of the bacilli. The ages varied from 23 months to 12 years.

(ase 1.-Willis $\mathrm{S}$., the youngeat child, 23 months, had an attack of diphtheria, moderately severe. One week later there was hoarseness followed by regurgitation of fluids through nose, nasal voice, and internal strabismus of both eyes. At the end of the first week the child was unable to stand alone, or walk, pain over track of nerves, most marked in the lower extremi ties, anesthesia, as shown by the indifference to prick of pins; the temperature test was not applied; the child lacked its normal intelligence. The symptoms increased rather in extent than intensity, the muscles of the neck becoming involved. The rectal temperature varied from 101 to 104.5 , with at the end 106. Death occurred in a spasm at the end of the fourth week from the first appearance of the sore throat.

Case 2.-Joe S., 4 years; seen on the eighth day of diphtheria. Klebs-Loeffler bacilli found, nose, pharynx and tonsils involved, moderately severe attack; throat normal and free from bacilli on the thirteenth day after the first symptoms. Thirtyone days after the first appearance of the sore throat, or eighteen days after recovery from the diphtheria, nasal voice and regurgitation of fluids were noticed, and the child walked with difficulty ; this increased in severity, the child became unable to either stand or walk alone, pain on pressure over the nerves of the lower extremity, with anesthesia. The muscles of the trunk and neck were not involved. The child again began to walk fifteen days after the symptoms of nasal voice and regurgitation were noticed, with complete recovery shortly after.

Case 3.- Sara N., had a mild attack of sore throat, accord ing to the history of the mother, from which she recovered. In the flfth week after, nasal voice and regurgitation of food began. The child complained of being tired and disinclined to walk; this gradually increased, and at the end of four weeks she was suddenly seized with pain in all the extremities, and shortly after was unable to stand, walk, sit or hold head up: marked tenderness and anesthesia in all extremities. The tem perature test showed great pain when heat was applied, but she insisted that the cold made her feel much better. The tenderness over the track of the nerves was intense; the tem. perature varied from 101 to 104.5 ; there was gradual loss of ntelligence. Five days after she complained of severe pain, the physical signs of broncho-pneumonia were found; death occurred in the ninth week from the first symptoms of sore throat.

Case 4.-John K., 3 years : recovered from a mild attack of chicken-pox about July 1 . On the 7 th the child was attacked by laryngeal diphtheria. Antitoxin was given, and the child was completely recovered by July 14 ; the bacilli persisted until July 31. A ugust 1 the mother noticed the nasal voice and a very elight regurgitation of fluids - on the 7th the child held the head to one side, staggered when attempting to walk, and fell down frequently; four days later was unable to stand alone; anesthesia marked; temperature test showed heat felt over a part of both limbs-of lower extremities, less on upper and on the trunk. Child at times seemed delirious, intelligence very much below his normal, died suddenly, apparently from cardiac paralysis on the eighteenth day after the first regurgitation of fluids.

Case 5.-Mild attack of diphtheria, December 30. Antitoxin given; complete recovery on the eighteenth day. February 7 , without previous reguritation of food, child began to limp, and fell down a great deal, complained of pain in the lege; four days later could walk only by dragging the feet, and at times crossed his legs during locomotion; could not stand nor walk alone; marked pain over track of nerves, with marked anesthesia of both legs; no reaction to cold temperature test. Slight reaction to heat; upper extremities normal ; could sit alone, but only a few minutes. On the sixteenth day internal strabismus of the left eye was noticed; on the twenty-first day attempted to walk; twenty days later completely well. The intelligence of this child was less affected than the others.

Case 6.-Annie P., 9 years. Early in November had an eruption on the hands, the nature of which was not known. No attention was paid to this, and about December 1 complained of inability to walk, and regurgitation of fluids and nasal voice. When seen a few days later could neither stand or sit alone; tenderness over track of nerves, patella reflex nearly absent. The symptoms remained about the same for a month, then marked change in the intelligence, utterly unable to move the lower limbs or hold the head up; tenderness over track of nerves much more marked, with marked anesthesia. Entire absence of patella reflex; slight atrophy of lower limbs; could not swallow even solid food without choking. All symptoms continued to increase in severity; intelligence became more affected ; there was anesthesia of all limbs and of the throat, as shown by the indifference to the introduction of the stomach tube, this being at first much resisted. The disease lasted from November 1, the time of appearance of the eruption, to January 29, when death occurred apparently from cardiac paralysis.

Case 7 -George Mc., 5 years. In the latter part of March had a mild sore throat; well in a week. One month later child began to behave strangely and staggered when walking. This slowly increased, then nasal voice and regurgitation of food. 
In ten days from first symptoms, child decidedly idiotic. Could not sit, stand or walk, even with much assistance. Pain over track of nerves, anesthesia most marked in the lower extremities. Patella reflex absent. On the fifteenth day very slight improvement, which gradually increased and the child was entirely well, with his usual intelligence thirty days later.

Case 8.-Charlie S., 4 years. Diphtheria September 1, moderately severe; treated with antitoxin; made a good recovery. October 5 child became sleepy, some regurgitation of fluids; two days later marked tenderness over track of nerves in the lower extremities; unable to stand alone or walk; four days after the first symptom of regurgitation, child unable to hold head up or sit alone; very marked tenderness over the track of the nerves in the lower extremity, to a less extent in the upper extremities; anesthesia marked over lower limbs, lese over upper; in parts of chest and abdomen marked anesthesia; much muco-purulent discharge in mouth and throat; intelligence much diminished; the lungs showed signs of a general broncho-pneumonia, and very numerous pleuritic râles. The patella reflex entirely absent. The anesthesia and paralysis became marked, and death occurred on the ninth day from the first symptom of regurgitation and weakness.

Case 9.-Christine N., 6 years. January 6 , mild attack of diphtheria, from which she apparently completely recovered. February 18, feverish and vomited; complained of much pain in both legs and was unable to walk; admitted to the New York Infirmary for Women and Children; absence of patella reflex on both sides; marked pain and tenderness over the track of the nerves, with very marked anesthesia to prick of pins and to the heat test; slight reaction to cold; upper limbs and trunk were normal; both feet extended; neither nasal voice or regurgitation of fluids; child not so bright as she is normally ; can not stand alone nor take a step alone. Symptoms gradually decreased and child was well, with normal sensation and no paralysis March 27.

Case 10.-Nettie B., 9 years. February 29, eruption of chickenpox, which followed a normal course. Two weeks later child was taken out by an elder sister and complained of being too tired to walk; was out a long time and returned complaining of pain in both legs and inability to stand or walk alone. When seen the next morning there was marked tenderness over the track of the nerves, diminished patella reflex. The inability to walk increased ; pain became more marked with noticeable anesthesia; nearly entire obliteration of patella reflex. Symptoms remained about the same; child could walk with great difficulty when supported. The disposition was extremely "cranky," the reverse of her normal condition. The upper extremities were at no time involved. At the end of three week the symptoms diminished in intensity, and gradual improvement took place. The child was perfectly well by May 15 .

Case 11.-Rose B. 11 years. Entered the New York Infirmary, February 20, with a mild attack of chorea; by March 22 there had been little improvement in the symptoms and 5 minims Fowler's solution was ordered to be given every three hours day and night, a minim to be added each day. On March 29 she was taking 11 minims, when she vomited and the drug was discontinued for twenty-four hours. April 7 , she was taking 119 minims, when a red papilla eruption was noticed, and symptoms of gastric catarrh, and the note was made that the arsenic had very little effect upon the chorea, so it was discontinued. April 13, the child seemed not to see and hear well. On the 19 th very little twitching; May 2, no twitching. During this time the child had boen disinclined to walk; had not seemed as intelligent as usual. May 12 the gait was very irregular and uncertain; dragged both legs; there was marked tenderness on pressure over the nerves; on the 21st utterly unable to stand alone, much pain over nerves, marked anesthesia in lower limbs. The symptoms of neuritis reached their height at this time and began slowly to disappear. June 4, began to walk a little with assistance; July 9 walking normally; no tenderness over the nerves; anesthesia had disappeared; discharged July 14, with no trace of either neuritis or chorea.

Several cases similar to Rose B. are found in medical literature, but the dose at the time of the development of the neuritis was smaller. This child received in the last twenty-four hours of the medication Fowler's solution fl 3 iiss, or $13 / 5$ gr. arsenious acid. One case is reported by Dr. Roberts, in which the child received 66 minims in twenty-four hours in three doses. In my case the symptoms of neuritis at first closely resembled those found at the end of chorea. The weakness and paralysis, with the anesthesia, appeared abruptly. The upper extremities were not affected, but the trunk muscle seemed weak; the child could sit alone only for a few moments.

Case 12.-Anna J., 4 years. December 2, eruption of measles in the normal manner. December 3 the child refused to speak ; seemed apathetic. A day or two later would smile and laugh without reason. Seven days after the first appearance of apathy the child could neither sit nor stand alone, cried when placed on her feet; marked tenderness over the track of the nerves in the lower extremities, with anesthesia. Between the third and the seventh day the symptoms gradually progressed and reached their height on the seventh day, remaining the same until the eleventh day, when she began to notice a little, and made her desires known by crying and pointing to objects, and attempted to stand but cried when placed on her feet. On the six teenth day made an attempt to speak, but seemed unable to articulate; tried to walk on the nineteenth day, and was able to articulate; could stand alone and walk, but incoordinately, on the twenty-second day; by the twenty-eighth day could speak distinctly, if not excited, put words into sentences, walked about but staggered some; pain over track of nerves and anesthesia nearly gone. Child walked normally at the end of six weeks; spoke as well as before illness at the end of three months.

In none of my cases were the sphincter muscles attacked. The family history in all cases was unim. portant, except in the case of George N., whose parents are alcoholic, and Charlie S., whose mother died of tuberculosis during the child's attack of diphtheria. All the children except Nettie B., were tenement-house children. The sanitary surroundings of John K. were especially bad; the child lived in rooms into which daylight came in midsummer only from 10 A.M. to 3 P.M.

The differential diagnosis was made chiefly from Landry's paralysis, but in this the paralysis begins in the legs, spreading from one to the other, then to the upper extremities; in my cases the paralysis began in both legs at the same time. In the cases of diphtheria the nasal voice and regurgitation preceded the loss of power in the limbs; in none of my cases was there a question of poliomyelitis. The question of Freidrich's disease, I think, could be thought of only in the case of the child with measles, the only resemblance being the disturbance of speech. From myelitis the diagnosis was made from the symmetrically located pain, and no involvement of either bladder or rectum, and in each case the distinct history of a preceding infectious disease, except in the case of Annie P., in which the diagnosis of an infectious disease was uncertain, but with the first symptoms of inability to walk, was present the regurgitation of fluids and nasal voice, which are not found in myelitis. The treatment con. sisted of strychnin, either by the mouth or hyper. dermatically; stimulants when indicated.

\section{THE RECOGNITION AND TREATMENT OF} EARLY MYXEDEMA IN CHILDHOOD.

Presented to the Section on Diseases of Children, at the Forty-ninth

Annual Meeting of the American Medical Association, held at Denver,Colo., June 7-10, 1898.

BY FREDERICK BIERHOFF, M.D.

Clinical Assistant, Department of Pediatrics, College of Physicians and Surgeons. NEW YORK CITY.

That myxedema is not a disease per se, but a symptom, or collection of symptoms, dependent upon the absence, or upon a diseased condition of the thyroid gland, is now generally accepted to be a fact, the degree of severity of the symptoms depending upon the extent to which destruction of the secreting portion of the gland in question has taken place. That cretinism, myxedema and goiter (so-called) are but 